\title{
Resveratrol suppresses colon cancer growth by targeting the AKT/STAT3 signaling pathway
}

\author{
DAN LI $^{1 *}$, GANGCHENG WANG $^{1 *}$, GUOGUO JIN $^{2 *}$, KE YAO $^{3}$, ZHENJIANG ZHAO $^{2}$, LIANGYU BIE ${ }^{4}$, \\ YONGJUN GUO ${ }^{5},{\text { NING } I^{4}, \text { WENYING DENG }}^{4}$, XIAOBIN CHEN $^{4}$, BEIBEI CHEN $^{4}$, \\ YUANYUAN LIU ${ }^{6}$, SUXIA LUO ${ }^{4}$ and ZHIPING GUO ${ }^{7}$ \\ ${ }^{1}$ Department of General Surgery, The Affiliated Cancer Hospital of Zhengzhou University, Zhengzhou, Henan 450003; \\ ${ }^{2}$ Laboratory of Bone Tumor, Henan Luoyang Orthopedic Hospital, Zhengzhou, Henan 450000; \\ ${ }^{3}$ China-US (Henan) Hormel Cancer Institute, Zhengzhou, Henan 450003; Departments of ${ }^{4}$ Oncology and \\ ${ }^{5}$ Molecular Pathology, The Affiliated Cancer Hospital of Zhengzhou University, Zhengzhou, Henan 450003; \\ ${ }^{6}$ Zhengzhou Central Hospital Affiliated to Zhengzhou University, Zhengzhou, Henan 450007; \\ ${ }^{7}$ Fu Wai Hua Zhong Vascular Disease Hospital, Zhengzhou, Henan 450018, P.R. China
}

Received May 9, 2018; Accepted October 30, 2018

DOI: $10.3892 /$ ijmm.2018.3969

\begin{abstract}
Colon cancer is a common type of cancer worldwide and accounts for a significant number of cancer-related deaths. Although surgical techniques and treatment strategies for colon cancer have advanced over the past two decades, the prognosis has not improved considerably. Resveratrol, a natural stilbene compound, possesses antioxidant, cardioprotective and anticancer properties. However, the role of resveratrol in colon cancer has not been fully elucidated. The present study demonstrated that resveratrol inhibited cell proliferation and colony growth in DLD1 and HCT15 colon cancer cells, but did not affect normal colon epithelial cells. The resveratrol-mediated inhibition of cell proliferation correlated with an induction of apoptosis and with $\mathrm{G}_{1}$ phase cell cycle arrest in colon cancer cells. Additionally, resveratrol treatment decreased the protein expression levels of cyclin D1, cyclin E2 and BCL2 apoptosis regulator, while it increased BCL2 associated $\mathrm{X}$ and tumor protein $\mathrm{p} 53$, all of which are involved in the regulation of cell cycle and apoptosis. Notably, the results obtained from in silico computational screening identified AKT serine/threonine kinase 1 (AKT1) and AKT2 as novel targets of resveratrol.
\end{abstract}

Correspondence to: Professor Suxia Luo, Department of Oncology, The Affiliated Cancer Hospital of Zhengzhou University, 127 Dongming Road, Zhengzhou, Henan 450003, P.R. China

E-mail: luosxrm@163.com

Professor Zhiping Guo, Fu Wai Hua Zhong Vascular Disease Hospital, 1 Fuwai Avenue, Zhengzhou, Henan 450018, P.R. China

E-mail: guozhiping74@hotmail.com

\section{*Contributed equally}

Key words: resveratrol, colon cancer, AKT serine/threonine kinase, signal transducer and activator of transcription 3, signaling pathway
Computational docking suggested that there are three or four possible hydrogen bonds in the active pocket of AKT1 and AKT2 that contribute to the mode of action of resveratrol. The present study confirmed that resveratrol bound to AKT1 and AKT2 with a pull-down assay. Furthermore, knockdown of AKT1 and AKT2 inhibited cell proliferation and colony growth, by attenuating cell cycle progression and increasing apoptosis in colon cancer cells, effects that were similar to those caused by resveratrol treatment. Taken together, the present results suggest that the targeting effects of resveratrol to AKT1 and AKT2 may be a potent strategy for chemoprevention or therapy for colon cancer.

\section{Introduction}

Colon cancer is the third most common malignancy and the fourth leading cause of cancer-related deaths worldwide $(1,2)$. In China, colon cancer is the 4-5th most commonly diagnosed type of cancer in men and women. Despite improvements in colon cancer diagnosis and therapy, many patients are still diagnosed at late stages, and the 5-year survival rate is only $30 \%$. Owing to its aggressive nature and poor response to chemotherapy, colon cancer remains a challenging disease to treat. Therefore, the identification and development of more effective drugs to prevent or treat colon cancer is urgently needed.

Over recent years, there has been increasing interest in natural compounds derived from plants that show potential preventative effects against cancer development but have minor side effects on normal cells or organs (3). Resveratrol (3,5,4'-trihydroxystilbene) is a natural compound derived from plants that can be found in large amounts in the skin of grapes and tomatoes as well as in red wine (4). It can also be extracted from the traditional Chinese plant Polygonum cuspidatum (5). Resveratrol has exhibited promising anticancer activity against breast cancer, ovarian cancer, leukemia, prostate cancer, liver cancer, melanoma, head and neck squamous cell carcinoma, thyroid cancer, and bladder cancer, via regulating 
cancer-related gene expression or cancer-associated signaling pathways (5-10). The anticarcinogenic action of resveratrol is associated to its ability to neutralize reactive oxygen species (ROS) and to modulate cellular processes, such as apoptosis and cancer cell proliferation and differentiation (11). Resveratrol can regulate the levels of ROS. Several papers have demonstrated that resveratrol increases ROS levels via extracellular signal-regulated kinase (ERK)1/2 inhibition and mitogen-activated protein kinase (MAPK) activation to induce human diffuse large B cell lymphoma cell apoptosis in vivo and in vitro (12). Resveratrol also regulates the activation of the mitochondrial enzyme system and the caspase cascade; upregulates the expression of cyclin-dependent kinase inhibitors, tumor suppressor genes, and death-induced cytokines and their receptors; and downregulates the expression of survival proteins associated with the development of chemoresistance, including survivin, cellular FLICE inhibitory protein (cFLIP), cellular inhibitor of apoptosis proteins (cIAPs), and antiapoptotic proteins BCL2 apoptosis regulator $(\mathrm{Bcl} 2)$ and BCL2 extra-large (Bcl-XL). Resveratrol activates AMP-activated protein kinase (AMPK) and inhibits the MAPK and phosphoinositide 3-kinase (PI3K)/AKT serine/threonine kinase (AKT) signaling pathways (13). It has also been reported that during the cancer promotion phase, resveratrol reduces the transcription and activity of cytochrome p450 (14). Several reports have illustrated that resveratrol inhibits the proliferation of colon cancer cells, induces colon cancer cell apoptosis and $\mathrm{G}_{2}$ phase arrest and modulates gene expression through its effects on p21 and BCL2 associated X (Bax) (10,15-17). However, the exact molecular mechanism requires further investigation.

AKT is a proto-oncogene belonging to the serine/threonine kinase family that regulates a large number of downstream mediators and ultimately controls critical cell survival and metabolic processes (18). In addition, it promotes cell cycle progression and inhibits apoptosis. AKT is highly activated in $60-70 \%$ of human colon cancers (19). The AKT signaling pathway is upregulated in numerous cancer types and is involved in cancer cell proliferation, survival, and metabolism (20). Two FDA-approved anticancer drugs, everolimus and temsirolimus, have employed targeted inhibition of AKT in the clinic and are currently in early clinical trials for various types of cancer $(21,22)$. Targeting AKT signaling, from the perspective of finding novel molecular targets for cancer therapy, has resulted in the discovery of candidates and their therapeutic inhibitors in crucial pathways. MK-2206, an AKT kinase inhibitor, has shown promising preclinical anticancer activity $(23,24)$ and entered phase II clinical trials for metastatic breast cancer and colorectal cancer in 2017. The crucial roles of AKT kinase have rendered it an attractive target for developing therapeutic cancer drugs (25). It has been reported that the AKT1 interaction with $\mathrm{N}$-ribosyldihydronicotinamide-quinone reductase (NQO2) is a target of resveratrol (26).

Signal transducer and activator of transcription (STAT)3 is an important transcription factor that translocates to the nucleus to regulate the expression of essential pro-invasive factors, such as matrix metallopeptidases, heat shock protein (HSP)70 and HSP90, and it is also downstream of various tyrosine kinase receptor signaling pathways that are involved in angiogenesis (27). Several reports have focused on the role of AKT phosphorylation and STAT3 translocation. Resveratrol can inhibit cell proliferation and promote cell apoptosis via the STAT3 signaling pathway $(28,29)$.

The present study investigated the anticancer activity of resveratrol in colon cancer cells. The results demonstrated that resveratrol inhibited cell growth of colorectal cancer by inhibiting AKT and its downstream signaling targets, and that AKT served as an upstream regulator of STAT3.

\section{Materials and methods}

Reagents. Resveratrol was purchased from Sigma-Aldrich (Merck KGaA, Darmstadt, Germany). Antibodies against phosphorylated (p-) STAT3 (Tyr705; cat. no. 9145), STAT3 (cat. no. 9139), p53 (cat. no. 48818), Bcl2 (cat. no. 15071), Bax (cat. no. 5023), AKT1 (cat. no. 75692), AKT2 (cat. no. 2964), cyclin D1 (cat. no. 2978) and cyclin E2 (cat. no. 4132) were purchased from Cell Signaling Technology, Inc. (Danvers, MA, USA). An AKT1/2 (cat. no. sc-1619) antibody was obtained from Santa Cruz Biotechnology, Inc. (Dallas, TX, USA). Active AKT1/2 recombinant protein was purchased from SignalChem (Richmond, BC, USA). RPMI-1640, fetal bovine serum (FBS) and Basal Medium Eagle (BME) were obtained from Biological Industries (Kibbutz Beit-Haemek, Israel). F-12K medium was purchased from Gibco (Thermo Fisher Scientific, Inc., Waltham, MA, USA).

Cell culture. The human colon cancer cell lines DLD1 and HCT15 were purchased from the American Type Culture Collection (Manassas, VA, USA). The human colonic epithelial cell (HCEC) line was kindly provided by Dr Jerry W Shay, University of Texas Southwestern Medical Center, Dallas, Texas (30). DLD1 and HCT15 cells were cultured in RPMI-1640 medium/10\% FBS. HCECs were cultured in F-12K medium $/ 10 \%$ FBS. All cells were cytogenetically tested and authenticated prior to freezing and were cultured with antibiotics at $37^{\circ} \mathrm{C}$ in a $5 \% \mathrm{CO}_{2}$ incubator for a maximum of 10 passages.

MTS assay. Cells at a density of $1 \times 10^{3}$ or $1 \times 10^{5}$ per well were seeded in 96-well plates in a final volume of $100 \mu 1$ per well for analysis of proliferation or cytotoxicity, respectively. Cells were cultured overnight and were then treated with different concentrations of resveratrol and incubated for various times, as indicated. Cell Titer96 Aqueous MTS reagent $(20 \mu \mathrm{l}$; Promega Corporation, Madison, WI, USA) was added to each well and the cells were incubated for an additional $1 \mathrm{~h}$ at $37^{\circ} \mathrm{C}$. The absorbance was then measured at $492 \mathrm{~nm}$ with a spectrophotometer (Multiskan; Thermo Fisher Scientific, Inc.).

Anchorage-independent cell growth assay. DLD1 or HCT15 cells $\left(8 \times 10^{3}\right.$ per well) were suspended in $1 \mathrm{ml}$ of BME/10\% FBS, and $0.33 \%$ agar with various concentrations of resveratrol and plated on a layer of solidified BME, $10 \% \mathrm{FBS}$, and $0.5 \%$ agar with the same concentration of resveratrol as the suspension. The cultures were maintained at $37^{\circ} \mathrm{C}$ in an incubator with $5 \% \mathrm{CO}_{2}$ for $1-2$ weeks. The colonies were photographed and counted with Image-Pro Plus software (v.6.2; Media Cybernetics, Rockville, MD, USA). 
Flow cytometry for analysis of apoptosis and cell cycle progression. DLD1 and HCT15 cells were seeded in 6-well plates and treated with different concentrations of resveratrol $(10,20,30$ or $40 \mu \mathrm{M})$ for $72 \mathrm{~h}$. The cells were harvested and stained with Annexin $\mathrm{V}$ and propidium iodide prior to flow cytometry analysis, data were analyzed by CellQuest Pro 6.0 (both from BD Biosciences, San Jose, CA, USA). For cell cycle detection, cells were fixed with ice-cold $70 \%$ ethanol overnight at $-20^{\circ} \mathrm{C}$. The cells were stained with propidium iodide, the cell cycle phase was determined by flow cytometry and data were analyzed by ModFit LTÔ 4.0.5 (Verity Software House, Inc., Topsham, ME, USA).

Western blot analysis. DLD1 and HCT15 cells were treated with various concentrations of resveratrol for $72 \mathrm{~h}$ and were then lysed with RIPA buffer (50 mM Tris base, 1\% NP-40, $0.25 \%$ sodium deoxycholate, $150 \mathrm{mM} \mathrm{NaCl}, 1 \mathrm{mM}$ EDTA and $0.1 \% \mathrm{SDS}$; dissolved in $400 \mathrm{ml}$ water and adjusted to $\mathrm{pH}$ 7.4) supplemented with $1 \mathrm{mM}$ PMSF. Protein concentration was determined with a bicinchoninic acid protein assay kit (Bio-Rad Laboratories, Inc., Hercules, CA, USA). Protein was loaded (50 $\mu \mathrm{g} /$ lane) and separated by $10 \%$ SDS-PAGE and then transferred onto polyvinylidene difluoride (PVDF) membranes (EMD Millipore Corp., Burlington, MA, USA). The membranes were blocked with $5 \%$ non-fat milk for $1 \mathrm{~h}$ at room temperature. The blots were then probed with the appropriate primary antibodies $(1: 1,000)$ overnight at $4^{\circ} \mathrm{C}$ and incubated with a horseradish peroxidase (HRP)-conjugated secondary antibody (1:3,000; cat. no. SC-2005; Santa Cruz Biotechnology, Inc.) at room temperature for $1 \mathrm{~h}$. Protein bands were visualized with a chemiluminescence reagent (GE Healthcare Biosciences, Pittsburgh, PA, USA).

Computational docking model. To further confirm that resveratrol can bind AKT1 and AKT2, a in silico docking assay was performed using the Schrödinger Suite 2017 software program (Schrödinger LLC, Cambridge, MA, USA). AKT1 (PDB ID, 3OCB) and AKT2 (PDB ID, 3D0E) crystal structures were first obtained from the Protein Data Bank and were then prepared under the standard procedures of the Protein Preparation Wizard (Schrödinger Suite 2017). Hydrogen atoms were added consistent with a $\mathrm{pH}$ of 7 , and all water molecules were removed. The ATP-binding site-based receptor grid of $\mathrm{AKT} 1 / 2$ was generated for docking. Resveratrol was prepared for docking with the default parameters using the LigPrep program. Then, the docking of resveratrol with AKT1 and AKT2 was accomplished with the default parameters under the extra precision mode of the program Glide. Finally, the best-docked representative structures were obtained.

In vitro pull-down assay. Resveratrol-Sepharose 4B beads (Amersham Pharmacia Biotech; GE Healthcare, Chicago, IL, USA) were prepared following the manufacturer's instructions. DLD1 and HCT15 cell lysates $(500 \mu \mathrm{g})$ were incubated with Resveratrol-Sepharose 4B beads with rocking overnight at $4^{\circ} \mathrm{C}$. Following incubation, the beads were washed 3 times with buffer (50 mM Tris- $\mathrm{HCl}$ pH 7.5, $5 \mathrm{mM}$ EDTA, $150 \mathrm{mM}$ $\mathrm{NaCl}, 1 \mathrm{mM}$ dithiothreitol, 0.01\% NP-40 and 0.2 mM PMSF). Bound proteins were analyzed by western blotting.
Preparation of AKT1/2 knockdown cells. The viral vectors and the packaging vectors (pMD2G, psPAX2, Mock, shAKT1 and shAKT2) were obtained from Sigma-Aldrich (Merck KGaA). Several shRNA sequences targeting AKT1 and AKT2 were tested. Then, all plasmids were transfected into 293T cells, and viral supernatant fractions were collected after $48 \mathrm{~h}$. DLD1 and HCT15 cells were infected with mock or shAKT1 and shAKT2 virus particles for $24 \mathrm{~h}$. Cells were selected with puromycin to obtain both AKT1- and AKT2-silenced cell lines (shAKT1/2). The appropriate experiments were performed with these cells until the control cells (without infection) completely died (usually 2-3 days) in the puromycin medium.

Statistical analysis. All quantitative data are presented as the mean values \pm standard deviation. Each experiment was repeated at least three times. Data were analyzed with SPSS 19.0 software (IBM Corporation, Armonk, NY, USA). Statistically significant differences were determined using one-way analysis of variance, and multiple comparisons between groups were conducted using the Dunnett's test. $\mathrm{P}<0.05$ was considered to indicate a statistically significant difference.

\section{Results}

Resveratrol suppresses the cell proliferation and colony growth of colon cancers. The chemical structure of resveratrol is shown in Fig. 1A. Resveratrol is a natural compound and has few side effects, giving it an important advantage. First, normal HCECs were treated with resveratrol. The results demonstrated that resveratrol had no toxicity in the normal HCECs until the $40 \mu \mathrm{M}$ concentration (Fig. 1B). However, resveratrol significantly decreased the growth of the DLD1 and HCT15 colon cancer cells when they were exposed to different concentrations (Fig. 1C). Furthermore, colony growth was also inhibited by treatment with resveratrol in a dose-dependent manner (Fig. 1D). These results demonstrated that resveratrol might be a potential treatment for colon cancer.

Resveratrol induces cell apoptosis and cell cycle arrest at the $G_{l}$ phase. Next, further analyses were conducted to determine whether resveratrol could lead to the inhibition of cancer cell growth by regulating cell cycle progression and apoptosis. The results of Annexin V staining revealed that resveratrol treatment resulted in a significant increase in the apoptosis rate in both cell lines, DLD1 and HCT15 (Fig. 2A). Cell cycle analysis revealed that resveratrol significantly induced cell cycle arrest at the $G_{1}$ phase (Fig. 2B). The effects of resveratrol were further verified by examining the expression levels of cell apoptosis and cell cycle biomarkers, using western blot analysis. The results revealed that resveratrol treatment decreased cyclin D1 and cyclin E1 (Fig. 2C), increased p53, and decreased $\mathrm{Bcl} 2$ protein expression levels in a dose-dependent manner (Fig. 2D).

Resveratrol binds with AKT1 and AKT2 kinases. To elucidate the underlying mechanism of resveratrol's effects, potential targets of resveratrol were screened by Schrödinger software (release 2017) (31). The results predicted that resveratrol may bind with AKT1 and AKT2. To elucidate the potential binding site, an in silico docking study was conducted 


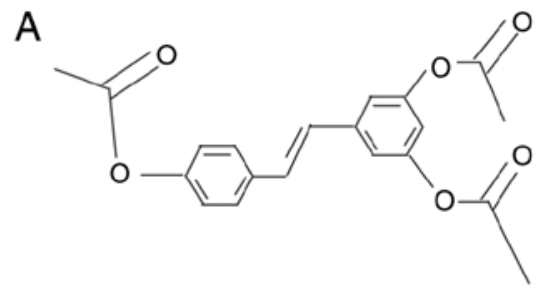

$\mathrm{B}$

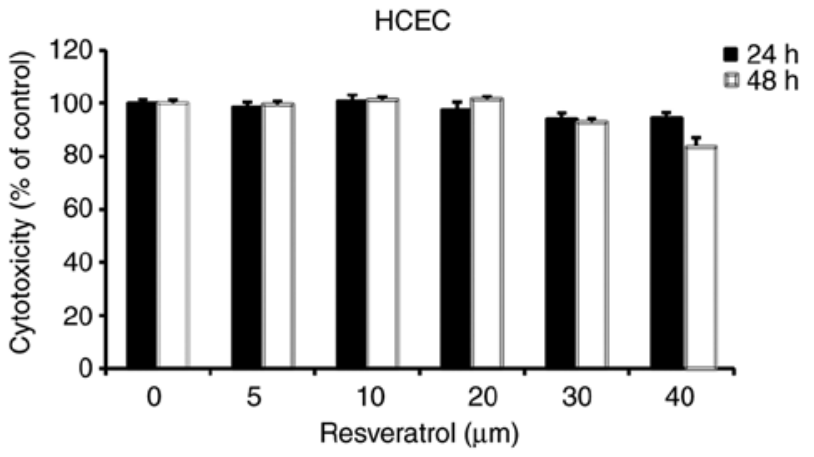

C

DLD1
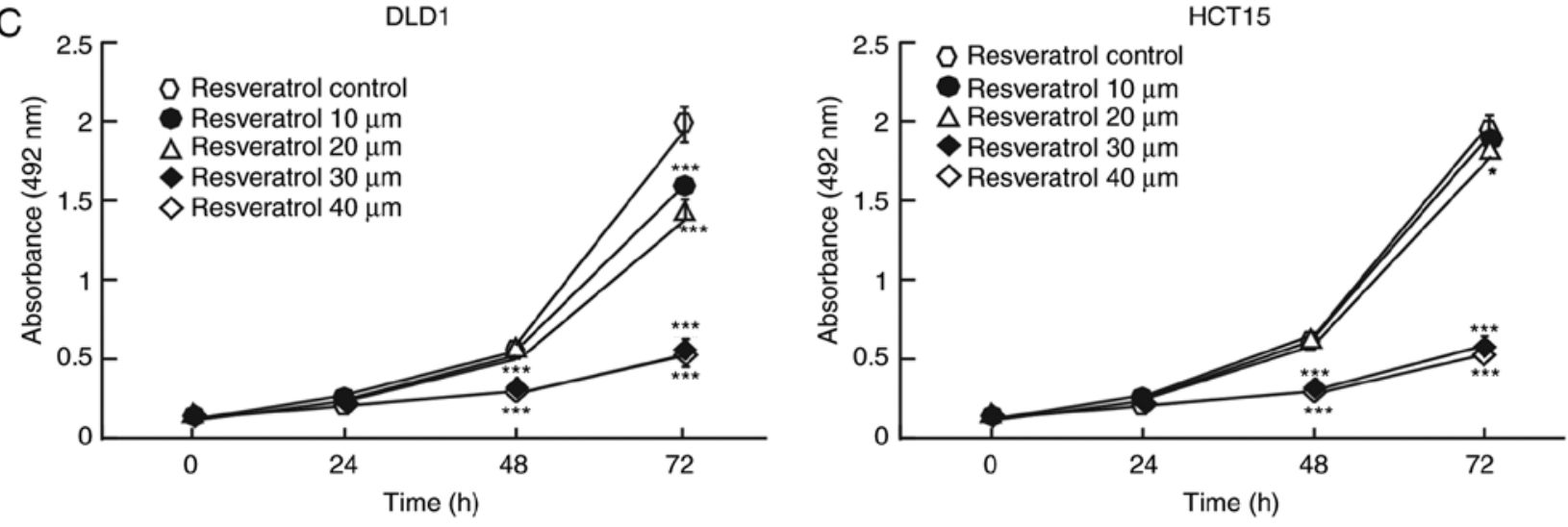

D

Resveratrol $(\mu \mathrm{m})$
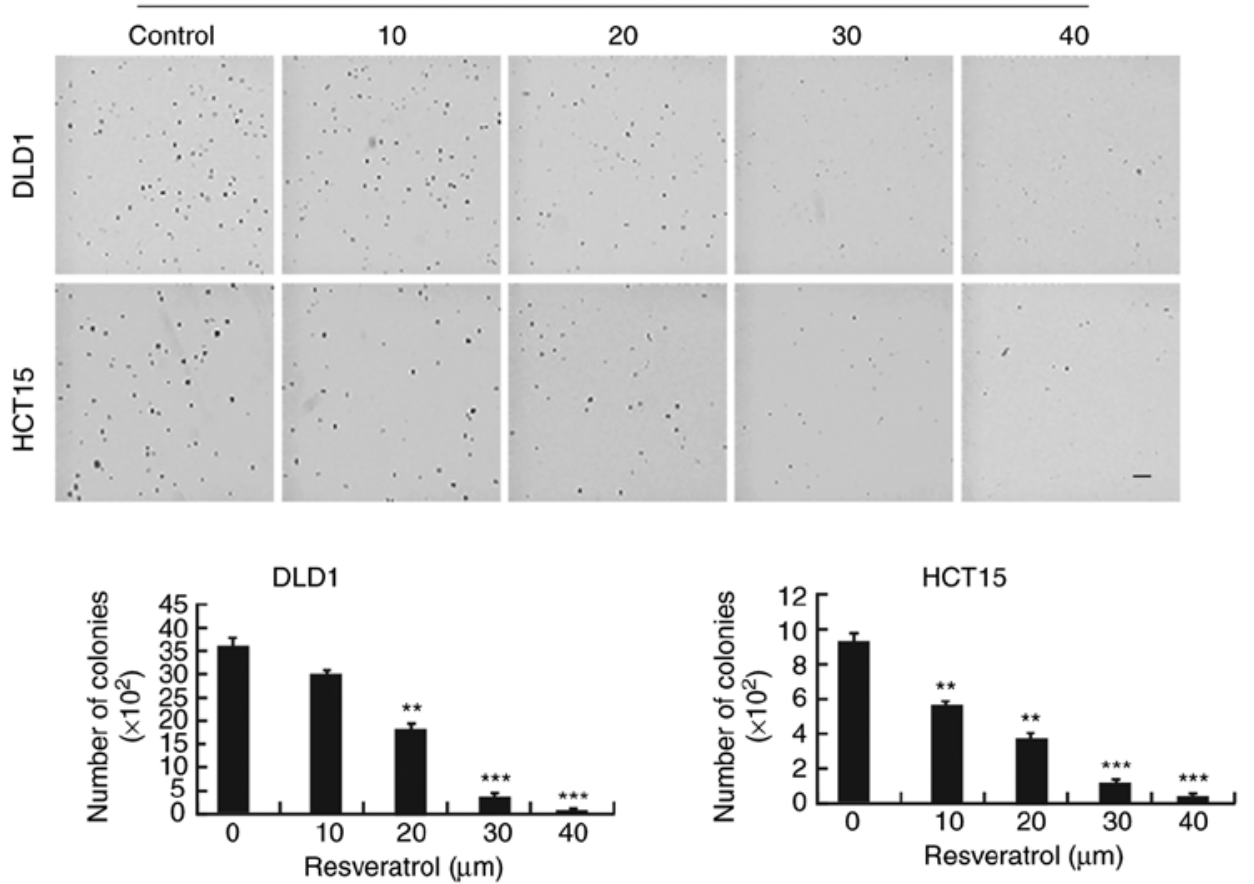

Figure 1. Effects of resveratrol on colon cancer cell proliferation and colony formation. (A) Chemical structure of resveratrol. (B) Toxicity of resveratrol in normal colon cells. HCECs were treated with various doses $(10,20,30$ or $40 \mu \mathrm{M})$ of resveratrol for $24 \mathrm{~h}$ or $48 \mathrm{~h}$, and toxicity was estimated with an MTS assay. (C) The effects of resveratrol on cell proliferation in the colon cancer cell lines DLD1 and HCT15. The cells were assessed at 24, 48 and 72 h with an MTS assay. (D) The effects of resveratrol on the anchorage-independent growth of DLD1 and HCT15 cells. Representative images are shown in the upper panel, and quantification graphs are shown in the lower panel. Scale bar, $100 \mu \mathrm{m} .{ }^{*} \mathrm{P}<0.05,{ }^{* *} \mathrm{P}<0.01$ and ${ }^{* * *} \mathrm{P}<0.001$ compared with untreated cells. HCEC, human colonic epithelial cell.

using the induced fit docking module in the Schrödinger software. The results indicated that resveratrol interacts with the ATP-binding pockets of AKT1 (Fig. 3A-a and b) and AKT2 (Fig. 3A-c and d). For the binding of resveratrol with
AKT1, the carbonyl oxygen of resveratrol interacts with the residues Glu228, Ala230 and Glu234 of AKT1 individually through hydrogen bonds (Fig. 3A-b). For the binding of resveratrol with AKT2, the carbonyl oxygen of resveratrol interacts 
A

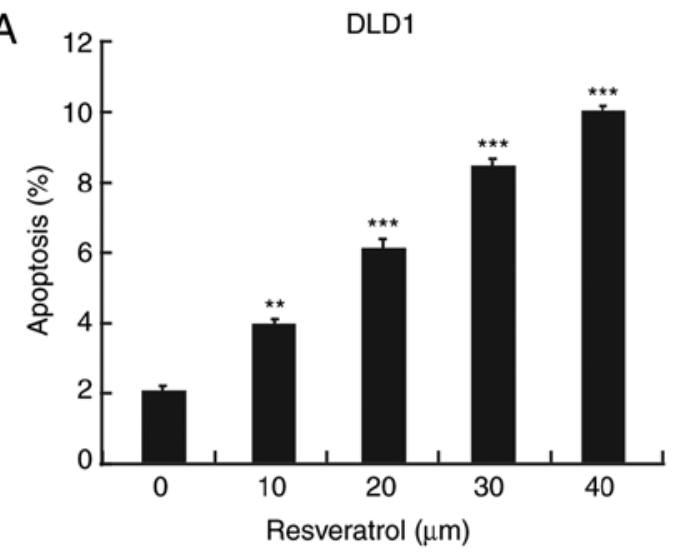

B

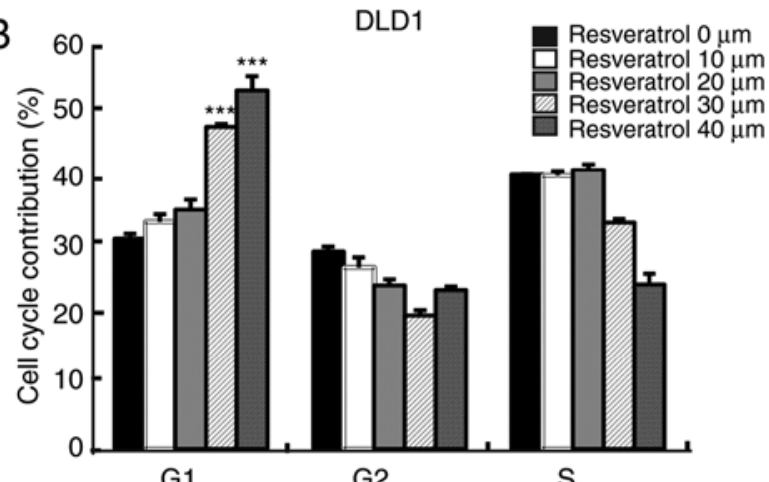

C

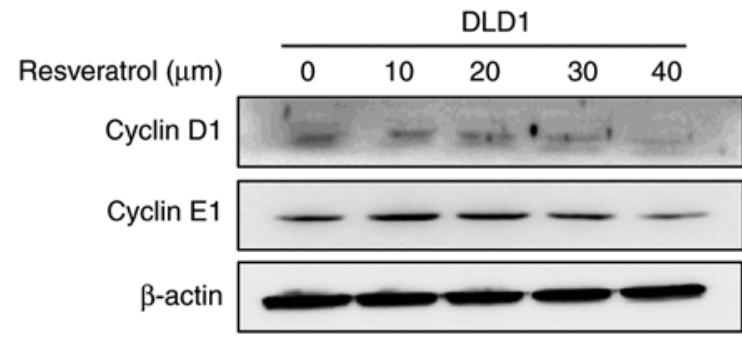

D

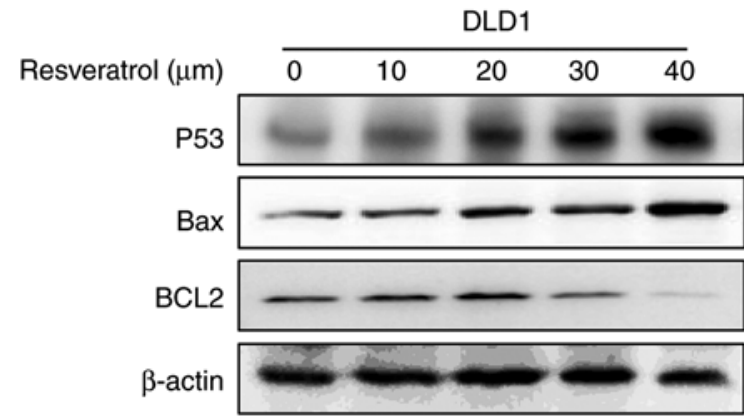

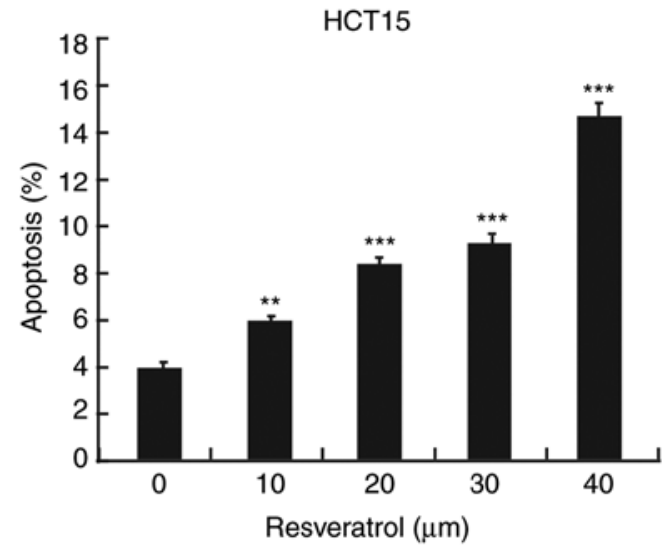
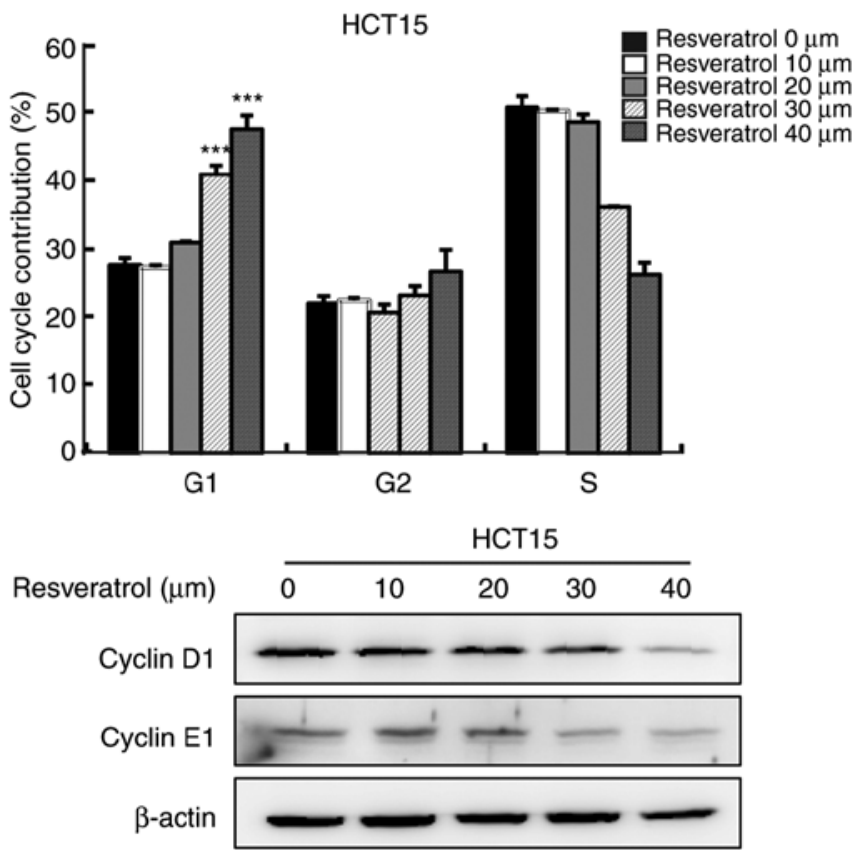

HCT15

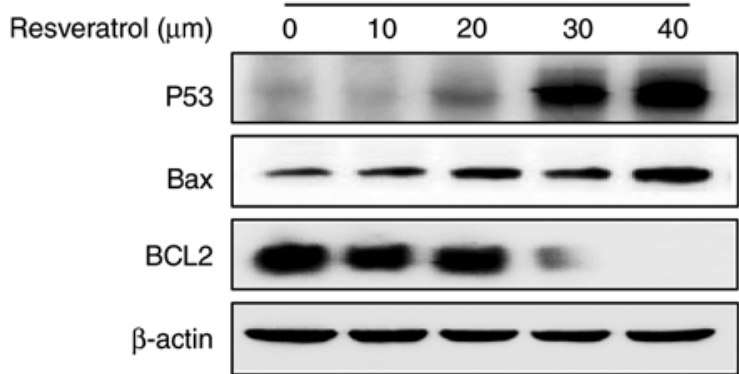

Figure 2. Resveratrol induces apoptosis and $\mathrm{G}_{1}$ phase cell cycle arrest in colon cancer cells. (A) Effects of resveratrol on cell apoptosis in the colon cancer cell lines DLD1 and HCT15. Cells treated with different concentrations of resveratrol for $72 \mathrm{~h}$ were analyzed with an Annexin V staining assay. (B) Cells treated with resveratrol had increased cell cycle arrest at the $\mathrm{G}_{1}$ phase. ${ }^{* *} \mathrm{P}<0.01$ and ${ }^{* * * *} \mathrm{P}<0.001$ compared with untreated cells. (C) Representative images from western blot analysis showing the effects of resveratrol on the expression of cyclin D1 and E2, and (D) on p53, Bax and Bcl2. The cells were treated with vehicle or resveratrol $(10,20,30$ or $40 \mu \mathrm{M})$. b-actin was used as the internal control. p53, tumor protein p53; Bax, BCL2 associated X; Bcl2, BCL2 apoptosis regulator.

with the residues Glu230, Ala232, Glu279 and Asp293 of AKT2 individually through hydrogen bonds (Fig. 3A-d). Thus, AKT1/2 activity might be dependent upon the presence of these residues. These computational results also indicated that resveratrol may elicit ATP-competitive inhibitory effects on the AKT1/2 kinase activity. To confirm the docking model, an in vitro pull-down assay was performed. The results demonstrated that Sepharose beads conjugated to resveratrol bound with AKT1 and AKT2 in both the DLD1 and the HCT15 cell lysates, but the Sepharose beads alone did not bind with AKT1 and AKT2 (Fig. 3B). Additionally, the expression of p-STAT3 (Tyr705), which is a downstream target, was suppressed by treatment with resveratrol in a dose-dependent manner (Fig. 3C). The total protein levels of STAT3 were not affected by treatment with resveratrol (Fig. 3C). These results illustrate that the AKT signaling 
A

a

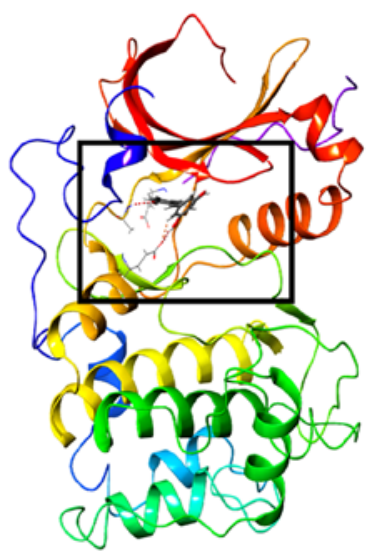

c

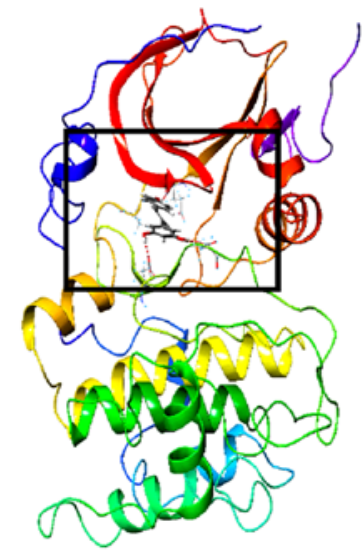

b

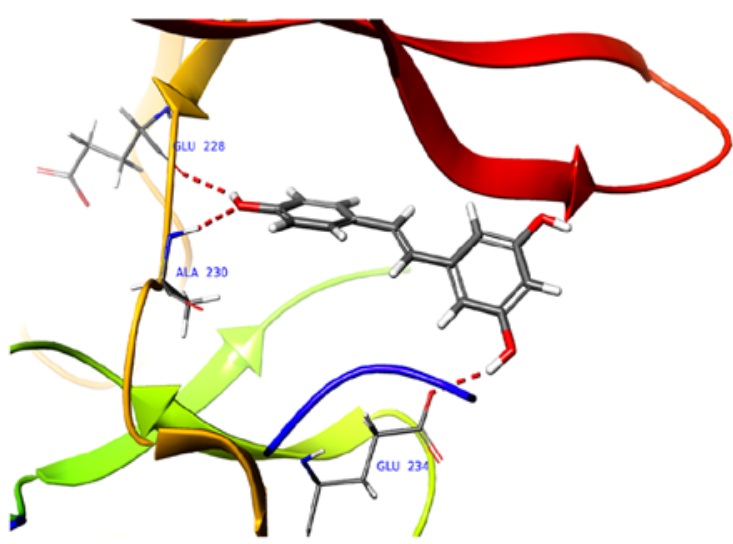

d

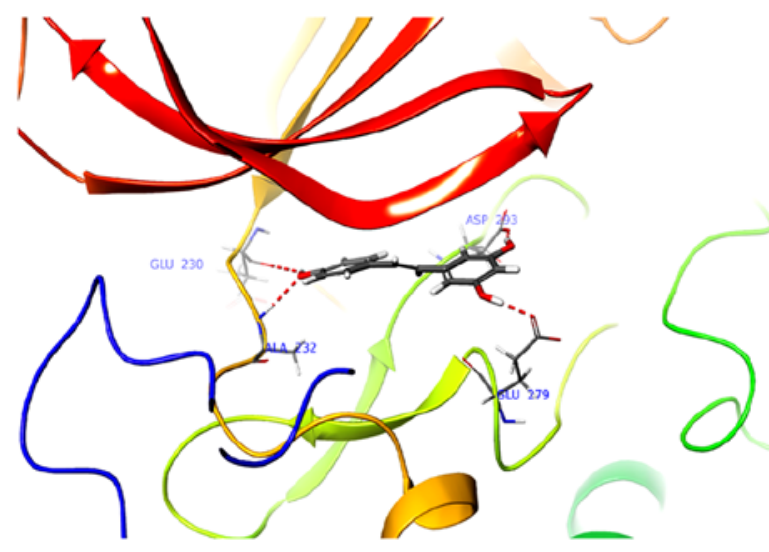

HCT15 cell lysate

C
Sepharose 4B

Resveratrol-Sepharose 4B
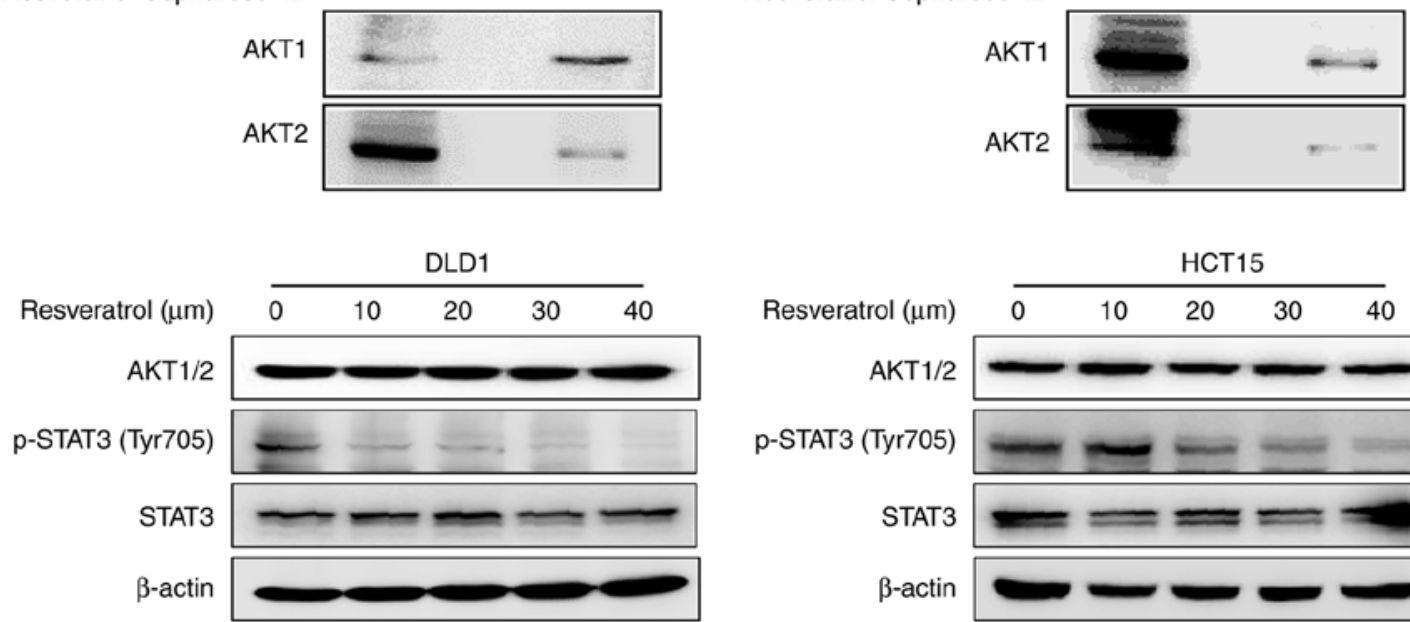

DLD1 cell lysate

Sepharose 4B Resveratrol-Sepharose 4B

DLD1

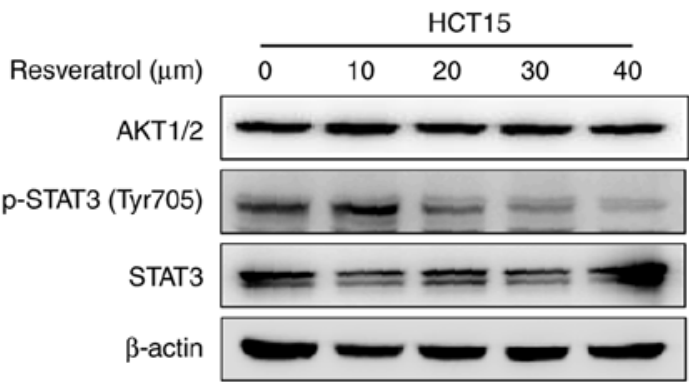

Figure 3. Resveratrol binds to AKT1 and AKT2. (A) Computational docking model of the binding between resveratrol and AKT1/2. (A-a) The binding sites of AKT1 are Ala230, Glu228 and Glu234 and (b) an enlarged view of the binding. (A-c) The binding sites of AKT2 are Ala232, Glu279, Glu230 and Asp293 and (d) an enlarged view of the binding. (B) Ex vivo pull-down assay between resveratrol and AKT1 or AKT2 with DLD1 and HCT15 cell lysates. (C) Representative images from western blot analysis of the expression of p-STAT3, total STAT3 and AKT1/2, following treatment with vehicle or resveratrol $(10,20,30$ or $40 \mu \mathrm{M})$. AKT, AKT serine/threonine kinase; STAT3, signal transducer and activator of transcription 3; p-, phosphorylated.

pathway is involved in the inhibitory effect of resveratrol in colon cancer cells.

AKT1/2 knockdown inhibits colon cancer cell proliferation and colony formation. Because of the crucial role of AKT in colon cancer, the present study examined whether knocking down AKT expression would produce an antitumor effect in colon cancer cells. After AKT1 and AKT2 were knocked down with short hairpin RNA (shRNA) in DLD1 and HCT15 colon cancer cells, the expression of AKT1 and AKT2 was markedly decreased (Fig. 4A). AKT1/2 knockdown markedly decreased the growth of both cell lines at 48 and $72 \mathrm{~h}$ (Fig. 4B). In addition, 
A

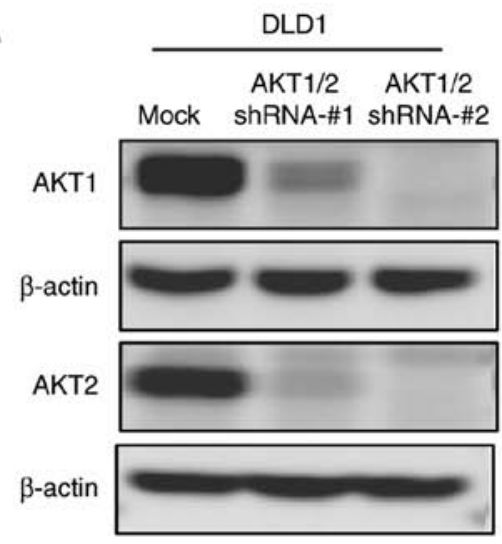

B

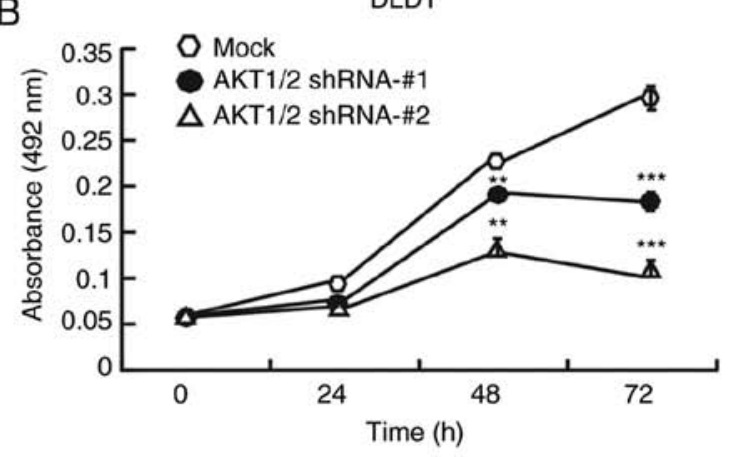

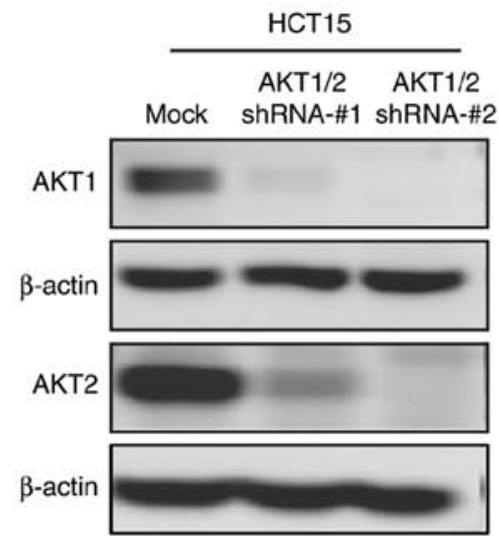

HCT15

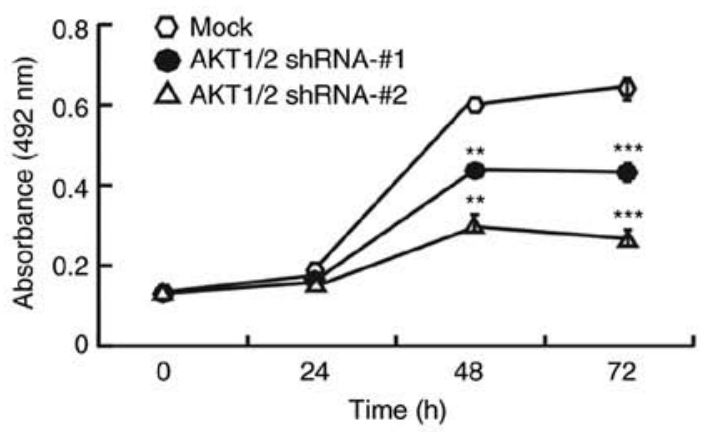

C

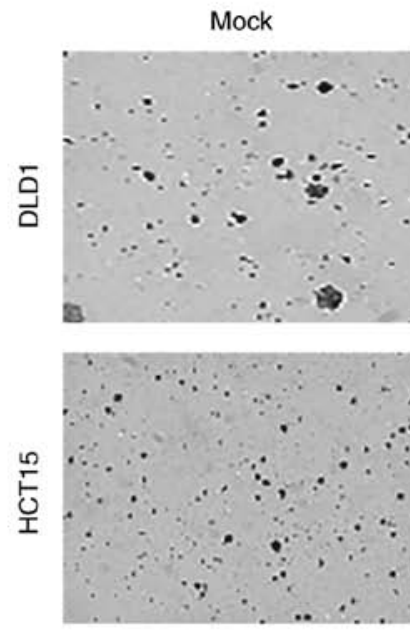

AKT1/2 shRNA-\#1

AKT1/2 shRNA-\#2
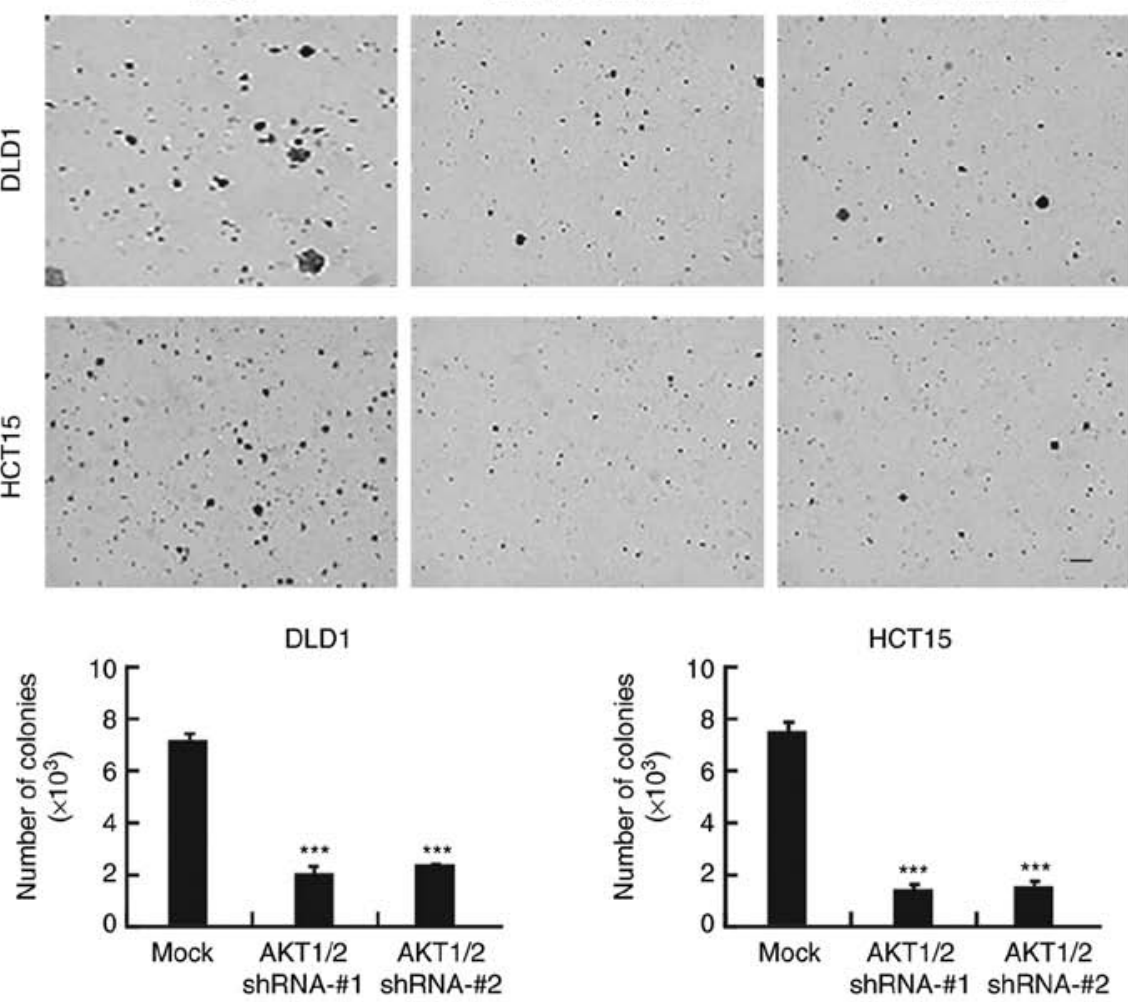

Figure 4. Roles of AKT1/2 in colon cancer cell growth. (A) Efficiency of AKT1/2 shRNA in DLD1 and HCT15 cells. (B) Effect of AKT1/2 knockdown on DLD1 and HCT15 cell proliferation and (C) colony formation (scale bar, $100 \mu \mathrm{m}$ ). ${ }^{* *} \mathrm{P}<0.01$ and ${ }^{* * *} \mathrm{P}<0.001$ compared with mock-treated cells. AKT, AKT serine/threonine kinase; shRNA, short hairpin RNA.

AKT1/2 knockdown exhibited a significant inhibitory effect on anchorage-independent colony growth by decreasing the number and size of colonies compared to the number and size of the colonies in the mock group (Fig. 4C).
Next, it was examined whether silencing AKT1/2 could affect cancer cell apoptosis or cell cycle progression. Upon AKT1/2 knockdown, the number of cells in the $G_{1}$ phase of the cell cycle increased, indicating that the cells suffered cell cycle 
A

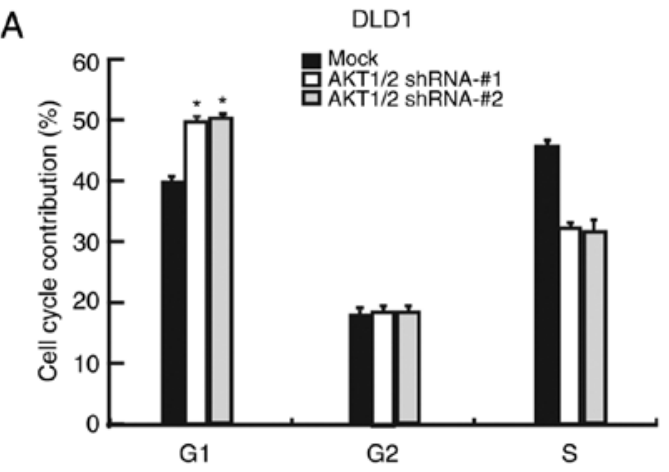

B

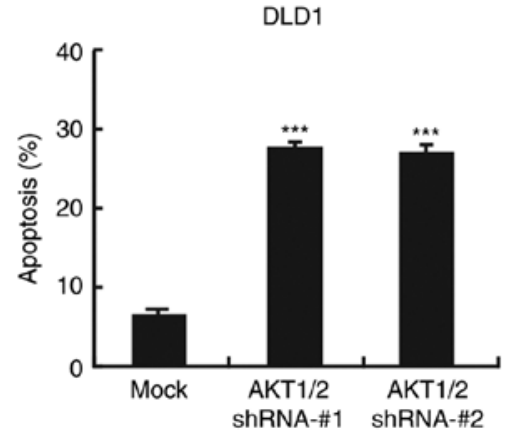

C

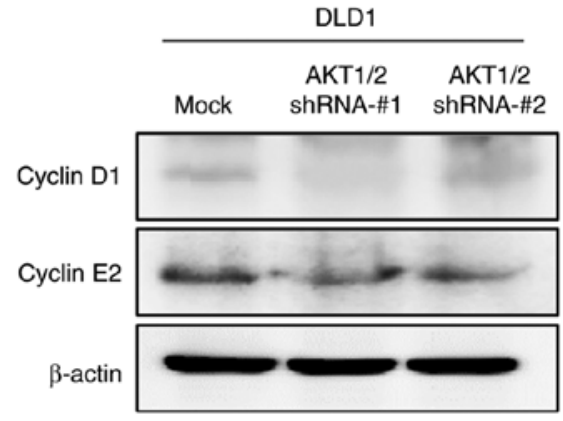

D

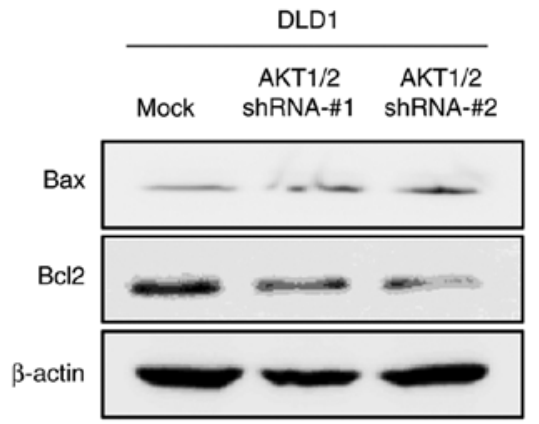

E

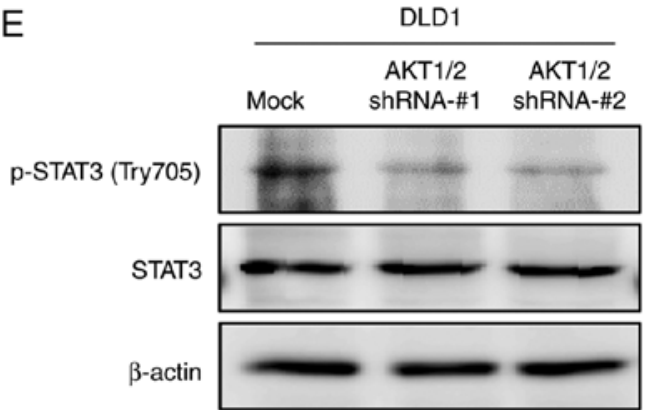

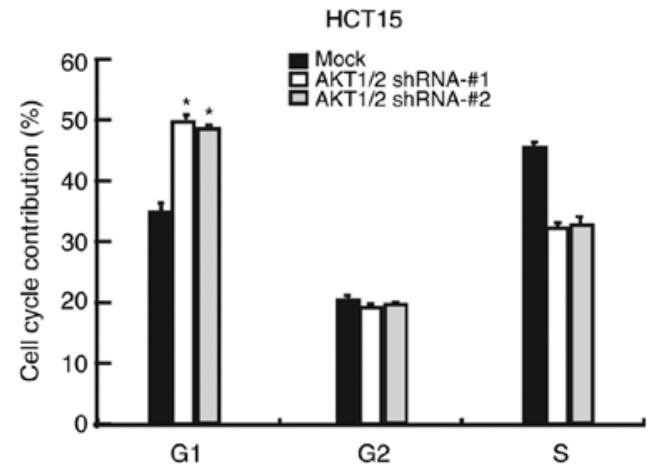
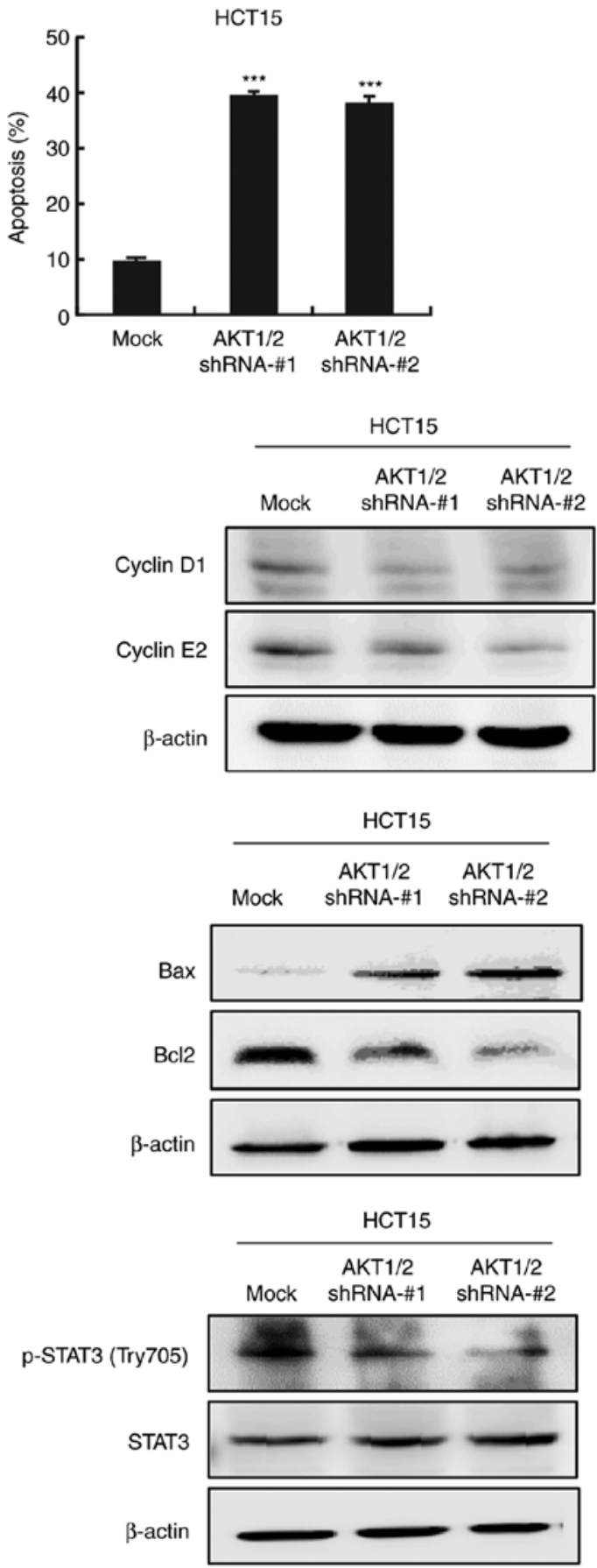

Figure 5. Effects of AKT1/2 knockdown on cellular processes. (A) Effect of AKT1/2 knockdown on DLD1 and HCT15 cell cycle progression and (B) apoptosis. " $\mathrm{P}<0.05$ and ${ }^{* * * *} \mathrm{P}<0.001$ compared with mock-treated cells. (C) Representative images from western blot analysis showing changes in the levels of cyclin D1 and cyclin E2 (cell cycle markers), (D) Bax and Bc12 (apoptosis markers), and (E) p-STAT3 and STAT3 (downstream signaling targets), following AKT1/2 knockdown. AKT, AKT serine/threonine kinase; Bax, BCL2 associated X; Bcl2, BCL2 apoptosis regulator; STAT3, signal transducer and activator of transcription 3; p-, phosphorylated; shRNA, short hairpin RNA. 


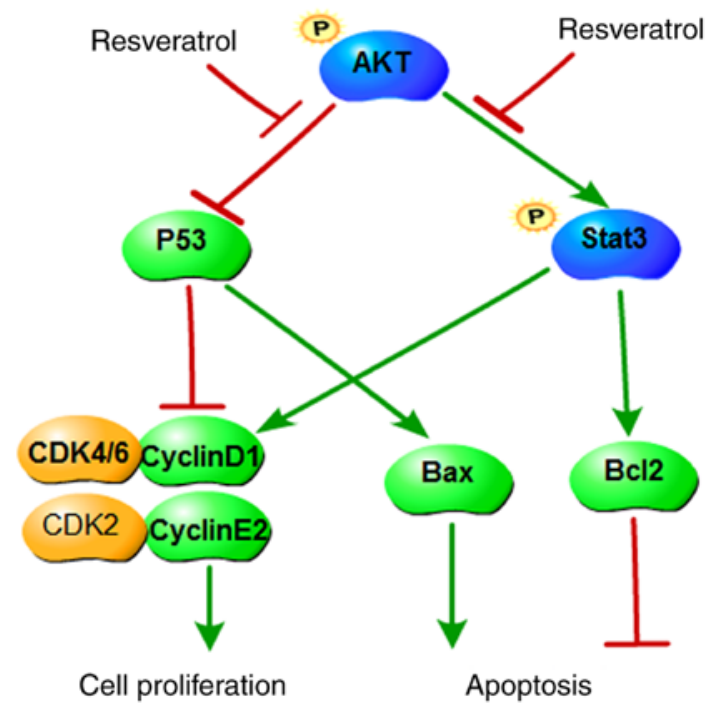

Figure 6. A proposed scheme illustrating the roles of AKT in colon cancer and its regulation by resveratrol. AKT upregulates $\mathrm{Bcl} 2$ and cyclin D1, promotes STAT3 phosphorylation, and downregulates p53 and Bax. Subsequently, AKT promotes cell cycle progression, prevents apoptosis and increases cell proliferation. Resveratrol inhibits the function of AKT and its downstream targets, therefore inducing cell cycle arrest and apoptosis, as well as inhibiting cell proliferation. AKT, AKT serine/threonine kinase; Bcl2, BCL2 apoptosis regulator; STAT3, signal transducer and activator of transcription 3; p53, tumor protein p53; Bax, BCL2 associated X; CDK, cyclin-dependent kinase.

arrest at the $\mathrm{G}_{1}$ phase (Fig. 5A). Furthermore, flow cytometry analysis revealed that the number of apoptotic cells increased following AKT1/2 silencing (Fig. 5B). The expression levels of cyclin D1 and cyclin E2, markers of the $\mathrm{G}_{1}$ phase, were markedly decreased following AKT1/2 silencing (Fig. 5C). In addition, Bax protein levels were increased, but $\mathrm{Bcl} 2$ protein levels were decreased in AKT1/2 knockdown cells, in both the DLD1 and HCT15 cell lines (Fig. 5D). Finally, STAT3 phosphorylation was also decreased in the AKT1/2 knockdown cells (Fig. 5E). These findings suggest that the AKT/STAT3 signaling pathway has an important role in colon cancer cells.

\section{Discussion}

Colorectal cancer (CRC) is the most common gastrointestinal tract cancer worldwide. Approximately 50\% of those diagnosed will succumb to colorectal cancer, making it the second leading cause of cancer-related deaths in both sexes. Despite decades of research and some promising discoveries, the mainstay of colorectal cancer treatment remains based on cytotoxic chemotherapy agents, such as irinotecan or oxaliplatin combined with fluoropyrimidine and leucovorin (FOLFIRI or FOLFOX6 regimens). Both the FOLFIRI and FOLFOX6 regimens have shown modest outcomes when used as first-line therapies. For colon cancer therapy, 5-fluorouracil (5-FU) and leucovorin have been the only options. The addition of irinotecan or oxaliplatin increases overall survival (OS) to 18 months. The present study demonstrated that resveratrol effectively inhibited the proliferation and colony formation of human colon cancer cells DLD1 and HCT15, and also induced cell apoptosis and $G_{1}$ phase cell cycle arrest. These findings suggested that resveratrol might be a promising cancer prevention agent or therapeutic agent. As a natural compound, resveratrol may have fewer side effects and lower toxicity to normal colon epithelial cells. Indeed, the present results demonstrated that resveratrol treatment had no effect in the viability of the normal HCEC cell line.

Multiple critical protein-encoding genes and pathways are believed to be responsible for tumorigenesis. Colorectal tumors contain a median of 76 mutations, and 15 of these affect candidate cancer genes. Increased understanding of the genetic and genomic changes in colorectal cancer has helped to direct therapies and predict responses. Genetic and epigenetic errors in signal transduction pathways result in malignant transformations and have thus emerged as key candidates for targeted molecular therapies. Over the past 10 years, the number of targeted agents used in various malignancies has increased dramatically. Currently, there are seven FDA-approved targeted agents for colorectal cancer, with many more in development and in clinical trials. The addition of targeted therapies over the past 10 years has improved OS in colorectal cancer by between 20 and 24 months. Resveratrol is a naturally occurring phytochemical that is produced by plants. Resveratrol has been demonstrated to have diverse biological properties, including anti-inflammatory, antioxidant, antiviral, neuroprotective, antifungal and anticancer properties $(32,33)$. Resveratrol has been reported to inhibit cancer cell growth via the AKT signaling pathway (34). The $\mathrm{PI} 3 \mathrm{~K} / \mathrm{AKT}$ pathway is an important endogenous protective mechanism that can prevent cell death and cell damage and is also associated with cancer progression and involved in drug resistance (35). AKT activation may lead to cell survival and can also inhibit apoptosis via the phosphorylation of BCL2 associated agonist of cell death (Bad) and caspase-9. STAT3 is an important transcription factor, and resveratrol can inhibit colon cancer cell proliferation via the STAT3 signaling pathway (36). Therefore, inhibition of this signaling pathway may be significant for cancer therapy. The computational docking results in the present study revealed that resveratrol interacted with the ATP-binding pockets of AKT1 and AKT2. In vitro pull-down assays confirmed that Sepharose beads conjugated to resveratrol bound AKT1 and AKT2 in colon cancer cell lysates. In addition, silencing AKT1/2 inhibited cell growth, increased apoptosis and induced $\mathrm{G}_{1}$ phase arrest. Several phase I clinical trials have investigated the potential of resveratrol for the treatment of patients with colon cancer (https://clinicaltrials.gov), performed by Nguyen et al (37), Patel et al (38) and Howells et al (39). The small sample size and the possible confounding effect of medications limited the conclusions reached; no definitive conclusions were obtained from any single trial. However, a set of well-designed and performed trials may provide more information, and this is a long lasting process.

In summary, the present study revealed that resveratrol inhibited colon cancer cell proliferation and colony formation, and induced cell apoptosis and $\mathrm{G}_{1}$ phase arrest. The mechanism of the anticancer effects of resveratrol was demonstrated to occur, at least partially, via inhibiting the AKT/STAT3 signaling pathway (Fig. 6). Taken together, the present results suggest that resveratrol may be a promising chemopreventive or therapeutic drug for colon cancer. Although resveratrol has a clear inhibitory effect on colon cancer cell proliferation and growth in vitro and ex vivo, these effects have not been 
confirmed yet in animal models and humans. Further research and clinical trials are warranted to fully elucidate the effects of resveratrol on human cancer.

\section{Acknowledgements}

Not applicable.

\section{Funding}

This study was supported by the Medical Science Research projects of Henan Province (grant no. 201702248), the Science and Technology Research Projects of Henan Province (grant no. 182102310376) and the Science and Technology Research Projects of Henan Province (grant no. 182102310125).

\section{Availability of data and materials}

The analyzed datasets generated during the study are available from the corresponding author on reasonable request.

\section{Authors' contributions}

DL, SL and ZG designed the study. GW and GJ performed the kinase and pull down assays. KY, ZZ, LB and YG analyzed the data. NL, WD, BC, YL and XC performed all other experiments. DL and GJ wrote the paper. All authors read and approved the final manuscript.

\section{Ethics approval and consent to participate}

Not applicable.

\section{Patient consent for publication}

Not applicable.

\section{Competing interests}

The authors declare that they have no competing interests.

\section{References}

1. Siegel RL, Miller KD and Jemal A: Cancer statistics, 2018. CA Cancer J Clin 68: 7-30, 2018.

2. Brenner H, Kloor M and Pox CP: Colorectal cancer. Lancet 383: 1490-1502, 2014.

3. Juan ME, Vinardell MP and Planas JM: The daily oral administration of high doses of trans-resveratrol to rats for 28 days is not harmful. J Nutr 132: 257-260, 2002.

4. Kundu JK and Surh YJ: Cancer chemopreventive and therapeutic potential of resveratrol: Mechanistic perspectives. Cancer Lett 269: 243-261, 2008.

5. Aluyen JK, Ton QN, Tran T, Yang AE, Gottlieb HB and Bellanger RA: Resveratrol: Potential as anticancer agent. J Dietary (Suppl 9): S45-S56, 2012.

6. Bai Y, Yang H, Zhang G, Hu L, Lei Y, Qin Y, Yang Y, Wang Q, Li R and Mao Q: Inhibitory effects of resveratrol on the adhesion, migration and invasion of human bladder cancer cells. Mol Med Rep 15: 885-889, 2017.

7. Rossi EL, Khatib SA, Doerstling SS, Bowers LW, Pruski M, Ford NA, Glickman RD, Niu M, Yang P, Cui Z, et al: Resveratrol inhibits obesity-associated adipose tissue dysfunction and tumor growth in a mouse model of postmenopausal claudin-low breast cancer. Mol Carcinog 57: 393-407, 2018.
8. Jiang Q, Yang M, Qu Z, Zhou J and Zhang Q: Resveratrol enhances anticancer effects of paclitaxel in HepG2 human liver cancer cells. BMC Complement Altern Med 17: 477, 2017.

9. Aggarwal BB, Bhardwaj A, Aggarwal RS, Seeram NP, Shishodia $S$ and Takada Y: Role of resveratrol in prevention and therapy of cancer: Preclinical and clinical studies. Anticancer Res 24: 2783-2840, 2004.

10. Zhou HB, Yan Y, Sun YN and Zhu JR: Resveratrol induces apoptosis in human esophageal carcinoma cells. World $\mathbf{J}$ Gastroenterol 9: 408-411, 2003.

11. Zheng X, Jia B, Song X, Kong QY, Wu ML, Qiu ZW, Li H and Liu J: Preventive potential of resveratrol in carcinogen-induced rat thyroid tumorigenesis. Nutrients 10: E279, 2018.

12. Jiang Z, Chen K, Cheng L, Yan B, Qian W, Cao J, Li J, Wu E, Ma Q and Yang W: Resveratrol and cancer treatment: Updates. Ann NY Acad Sci 1403: 59-69, 2017.

13. Liu YZ, Wu K, Huang J, Liu Y, Wang X, Meng ZJ, Yuan SX, Wang DX, Luo JY, Zuo GW, et al: The PTEN/PI3K/Akt and $\mathrm{Wnt} / \beta$-catenin signaling pathways are involved in the inhibitory effect of resveratrol on human colon cancer cell proliferation. Int J Oncol 45: 104-112, 2014.

14. Saiko P, Szakmary A, Jaeger W and Szekeres T: Resveratrol and its analogs: Defense against cancer, coronary disease and neurodegenerative maladies or just a fad? Mutat Res 658: 68-94, 2008.

15. Mahyar-Roemer M, Katsen A, Mestres P and Roemer K: Resveratrol induces colon tumor cell apoptosis independently of p53 and precede by epithelial differentiation, mitochondrial proliferation and membrane potential collapse. Int J Cancer 94: 615-622, 2001.

16. Malhotra A, Bath S and Elbarbry F: An organ system approach to explore the antioxidative, anti-inflammatory, and cytoprotective actions of resveratrol. Oxid Med Cell Longev 2015: 803971, 2015.

17. Tessitore L, Davit A, Sarotto I and Caderni G: Resveratrol depresses the growth of colorectal aberrant crypt foci by affecting bax and p21(CIP) expression. Carcinogenesis 21: 1619-1622, 2000.

18. Cheaib B, Auguste A and Leary A: The PI3K/Akt/mTOR pathway in ovarian cancer: Therapeutic opportunities and challenges. Chin J Cancer 34: 4-16, 2015.

19. Colakoglu T, Yildirim S, Kayaselcuk F, Nursal TZ, Ezer A, Noyan T, Karakayali H and Haberal M: Clinicopathological significance of PTEN loss and the phosphoinositide 3-kinase/Akt pathway in sporadic colorectal neoplasms: Is PTEN loss predictor of local recurrence? Am J Surg 195: 719-725, 2008.

20. Fresno Vara JA, Casado E, de Castro J, Cejas P, Belda-Iniesta C and González-Barón M: PI3K/Akt signalling pathway and cancer. Cancer Treat Rev 30: 193-204, 2004.

21. Polivka J Jr and Janku F: Molecular targets for cancer therapy in the PI3K/AKT/mTOR pathway. Pharmacol Ther 142: 164-175, 2014.

22. Fumarola C, Bonelli MA, Petronini PG and Alfieri RR: Targeting PI3K/AKT/mTOR pathway in non small cell lung cancer. Biochem Pharmacol 90: 197-207, 2014.

23. Agarwal E, Chaudhuri A, Leiphrakpam PD, Haferbier KL, Brattain MG and Chowdhury S: Akt inhibitor MK-2206 promotes anti-tumor activity and cell death by modulation of AIF and Ezrin in colorectal cancer. BMC Cancer 14: 145, 2014.

24. Sun Z, Wang Z, Liu X and Wang D: New development of inhibitors targeting the $\mathrm{PI} 3 \mathrm{~K} / \mathrm{AKT} / \mathrm{mTOR}$ pathway in personalized treatment of non-small-cell lung cancer. Anticancer Drugs 26: $1-14,2015$.

25. Pal I and Mandal M: PI3K and Akt as molecular targets for cancer therapy: Current clinical outcomes. Acta Pharmacol Sin 33: 1441-1458, 2012.

26. Hsieh TC, Lin CY, Bennett DJ, Wu E and Wu JM: Biochemical and cellular evidence demonstrating AKT-1 as a binding partner for resveratrol targeting protein NQO2. PLoS One 9: e101070, 2014.

27. Neradugomma NK, Subramaniam D, Tawfik OW, Goffin V, Kumar TR, Jensen RA and Anant S: Prolactin signaling enhances colon cancer stemness by modulating Notch signaling in a Jak2-STAT3/ERK manner. Carcinogenesis 35: 795-806, 2014.

28. Kang FB, Wang L, Jia HC, Li D, Li HJ, Zhang YG and Sun DX: B7-H3 promotes aggression and invasion of hepatocellular carcinoma by targeting epithelial-to-mesenchymal transition via JAK2/STAT3/Slug signaling pathway. Cancer Cell Int 15: 45, 2015.

29. Turkson J: STAT proteins as novel targets for cancer drug discovery. Expert Opin Ther Targets 8: 409-422, 2004. 
30. Roig AI, Eskiocak U, Hight SK, Kim SB, Delgado O, Souza RF Spechler SJ, Wright WE and Shay JW: Immortalized epithelial cells derived from human colon biopsies express stem cell markers and differentiate in vitro. Gastroenterology 138: 1012-1021 e1011-e1015, 2010.

31. Schrödinger Suite 2017. Schrödinger LLC, New York, NY, 2017.

32. Vang O: Resveratrol: Challenges in analyzing its biological effects. Ann NY Acad Sci 1348: 161-170, 2015.

33. Carter LG, D'Orazio JA and Pearson KJ: Resveratrol and cancer: Focus on in vivo evidence. Endocr Relat Cancer 21: R209-R225, 2014.

34. Kim SY, Hyun MY, Go KC, Zouboulis CC and Kim BJ: Resveratrol exerts growth inhibitory effects on human SZ95 sebocytes through the inactivation of the PI3-K/Akt pathway. Int J Mol Med 35: 1042-1050, 2015.

35. Yang F, Gao JY, Chen H, Du ZH, Zhang XQ and Gao W: Targeted inhibition of the phosphoinositide 3-kinase impairs cell proliferation, survival, and invasion in colon cancer. Onco Targets Ther 10: 4413-4422, 2017.
36. Li Y, Zhu W, Li J, Liu M and Wei M: Resveratrol suppresses the STAT3 signaling pathway and inhibits proliferation of high glucose-exposed HepG2 cells partly through SIRT1. Oncol Rep 30: 2820-2828, 2013.

37. Nguyen AV, Martinez M, Stamos MJ, Moyer MP, Planutis K, Hope $\mathrm{C}$ and Holcombe RF: Results of a phase I pilot clinical trial examining the effect of plant-derived resveratrol and grape powder on Wnt pathway target gene expression in colonic mucosa and colon cancer. Cancer Manag Res 1: 25-37, 2009.

38. Patel KR, Brown VA, Jones DJ, Britton RG, Hemingway D, Miller AS, West KP, Booth TD, Perloff M, Crowell JA, et al: Clinical pharmacology of resveratrol and its metabolites in colorectal cancer patients. Cancer Res 70: 7392-7399, 2010

39. Howells LM, Berry DP, Elliott PJ, Jacobson EW, Hoffmann E, Hegarty B, Brown K, Steward WP and Gescher AJ: Phase I randomized, double-blind pilot study of micronized resveratrol (SRT501) in patients with hepatic metastases-safety, pharmacokinetics, and pharmacodynamics. Cancer Prev Res 4: 1419-1425, 2011. 